Supporting information

\title{
Demonstration of superior Deep-UV Surface-Enhanced Resonance Raman Scattering (SERRS) substrate and single-base mutation detection in Oligonucleotides
}

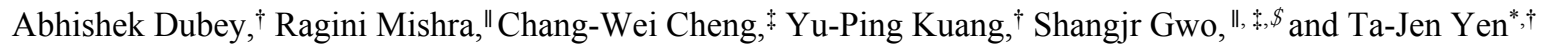 \\ ${ }^{\dagger}$ Department of Materials Science and Engineering, National Tsing Hua University, Hsinchu 30013, Taiwan \\ "Institute of Nanoengineering and Microsystems, National Tsing Hua University, Hsinchu 30013, Taiwan \\ ॠDepartment of Physics, National Tsing Hua University, Hsinchu 30013, Taiwan \\ \$Research Centre for Applied Sciences, Academia Sinica, Nankang, Taipei 11529, Taiwan
}

KEYWORDS : Surface-enhanced resonance Raman scattering, Aluminum plasmonics, Deep-UV Raman, Mutation 


\section{Supporting Note 1. Growth and Fabrication of Al nanohole array.}

Epitaxial Al film is grown by plasma assisted molecular beam epitaxy method on 2" c-plane sapphire substrate. After growth of Al film, it is cleaned by IPA, Ethanol, and DI water. Firstly, PMMA photoresist is coated on the $\mathrm{Al}$ film and nanohole array pattern is designed using electron beam lithography (ELS-7500). Further, $\mathrm{Al}$ film is etched by $\mathrm{Cl}_{2}$ and $\mathrm{BCl}_{3}$ using TCP-RIE (Transformer coupled Plasma-reactive ion etching) method. The thickness of the native oxide on the epitaxial $\mathrm{Al}$ approximates $\sim 3.5 \mathrm{~nm}$. This oxide thickness is also considered during the simulation methods. ${ }^{1}$

\section{Supporting Note 2. Reflectance and Raman Measurement}

A home built combined optical setup is used for reflectance and DUV Raman measurements. A fiber-coupled LDLS white light broad band source $(190 \mathrm{~nm}-2100 \mathrm{~nm})$ is used for realization of reflectance behavior of Al nanohole and FQCW (CryLas) $266 \mathrm{~nm}$ Laser is used for Raman excitation. The reflectance and Raman measurements are carried out using backscattering configuration. The back scattered signals for reflectance are collected through 40X DUV (LMU 40X -UVB, Thor lab) and recorded at Ocean optics spectrometer (HR4Pro). The Raman signals are collected through 77X reflective objective lens and recorded at $1800 \mathrm{~g} / \mathrm{mm}$ based spectrometer (Horiba Jobin Yvon FHR640). The detailed optical setup is shown in the figure S2.

\section{Supporting Note 3 . Sublimation of the bases of oligonucleotides}

For the performance of surface-enhanced resonance Raman scattering (SERRS), the monomers Adenine, Thymine, Guanine, and Cytosine were purchased from Sigma Aldrich and sublimated on the desire substrate under high vacuum $\left(\sim 1 \times 10^{6}\right)$ using an organic vacuum deposition system. The thickness was monitored using a quartz crystal monitor. The sublimation temperature of 
adenine, thymine, cytosine, and guanine is approximate $180{ }^{\circ} \mathrm{C}, 250{ }^{\circ} \mathrm{C}, 190{ }^{\circ} \mathrm{C}$, and $160{ }^{\circ} \mathrm{C}$ respectively.

\section{Supporting Note 4. ss-DNA functionalization}

All the standard customized ss-DNA oligonucleotides were purchased from QIAGEN with ultrapurification. DNA solutions were prepared using ultra-pure biological grade DI water. $10 \mu \mathrm{M}$ aqueous solution of ss-DNA was used for all the experiments. The ss-DNA solution was drop cast on the Al nanohole pattern/quartz and dried under vacuum.

\section{Supporting Note 5. Enhancement Factor}

The enhancement factor for SERRS substrate is calculated using the following equation:

$$
\text { Enhancement Factor }=\frac{\mathrm{I}_{\text {SERRS }} / \mathrm{N}_{\text {SERRS }}}{\mathrm{I}_{\mathrm{RRS}} / \mathrm{N}_{\mathrm{RRS}}}
$$

Where $\mathrm{N}_{\text {SERRS }}$ and $\mathrm{N}_{\text {RRS }}$ are the number of molecules contributing to the SERRS and Resonance Raman scattering (RRS) signal intensity. $\mathrm{I}_{\text {SERRS }}$ and $\mathrm{I}_{\text {RRS }}$ are the SERRS and RRS signal intensities, respectively.

Table S1 : The enhancement factor of DUV-SERRS substrate

\begin{tabular}{|c|c|c|c|}
\hline & Materials & Nucleotides & $(\sim \mathrm{EF})$ \\
\hline This work & Epitaxial Al nanohole array & $\mathrm{A}, \mathrm{T}, \mathrm{C}, \mathrm{G}$, and $\mathrm{U}$ & $\sim 10^{6}$ \\
\hline S.K. Jha et al. ${ }^{2}$ & Al nanoparticle & $\mathrm{A}$ & $\sim 10^{4}$ \\
\hline D.O. Sigle et al. ${ }^{3}$ & Al nanovoids & A & $\sim 10^{2}$ \\
\hline Tao Ding et al. ${ }^{4}$ & Al nanovoids & $\mathrm{A}$ & $\sim 10^{3}$ \\
\hline Y. Kumamoto et al. ${ }^{5}$ & Indium nanoparticle & A & $\sim 10^{2}$ \\
\hline B. Sharma et al. ${ }^{6}$ & Al film-over-nanosphere & $\mathrm{A}$ & $\sim 10^{6}$ \\
\hline
\end{tabular}


Supporting Note 6. Table S2 : The sequence of used ss-DNA

\begin{tabular}{|c|c|}
\hline ss-DNA name & sequence \\
\hline$(\mathrm{A})_{12}$ & AAAAAAAAAAAA \\
\hline$(\mathrm{C})_{12}$ & CCCCCCCCCCCC \\
\hline$(\mathrm{T})_{12}$ & TTTTTTTTTTTT \\
\hline$(\mathrm{G})_{12}$ & GGGGGGGGGGG \\
\hline $\mathrm{A}_{1} \mathrm{C}_{11}$ & ACCCCCCCCCCC \\
\hline $\mathrm{A}_{3} \mathrm{C}_{9}$ & AAACCCCCCCCC \\
\hline $\mathrm{A}_{5} \mathrm{C}_{7}$ & AAAAACCCCCCC \\
\hline $\mathrm{A}_{7} \mathrm{C}_{5}$ & AAAAAAACCCCC \\
\hline $\mathrm{A}_{9} \mathrm{C}_{3}$ & AAAAAAAAACCC \\
\hline $\mathrm{A}_{11} \mathrm{C}_{1}$ & AAAAAAAAAAAC \\
\hline
\end{tabular}


a

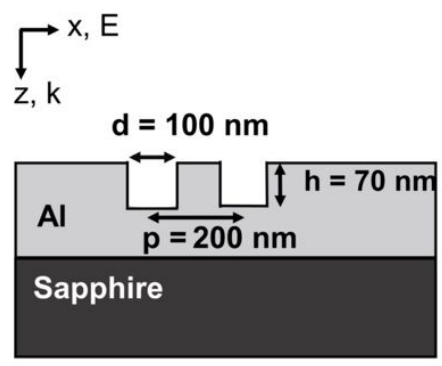

b

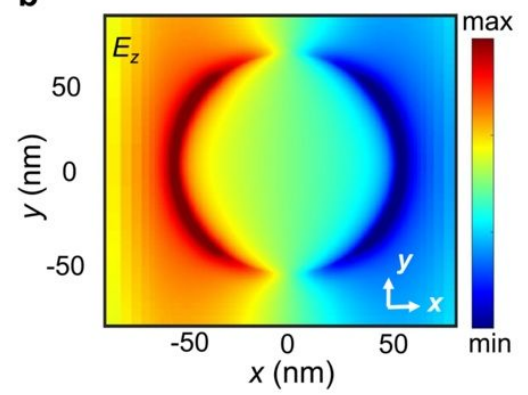

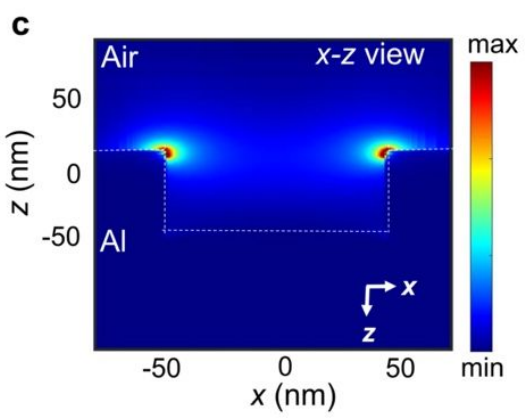

Figure S1 a, The schematic outline of parameter of DUV SERRS substrate. b, The x-y view of $E_{z}$ component of electric field in Al nanohole which confirms also the formation of dipole at $266 \mathrm{~nm}$. c, The $\mathrm{x}-\mathrm{Z}$ view of electric field confinement in Al nanohole at $266 \mathrm{~nm}$ using FDTD simulation method.

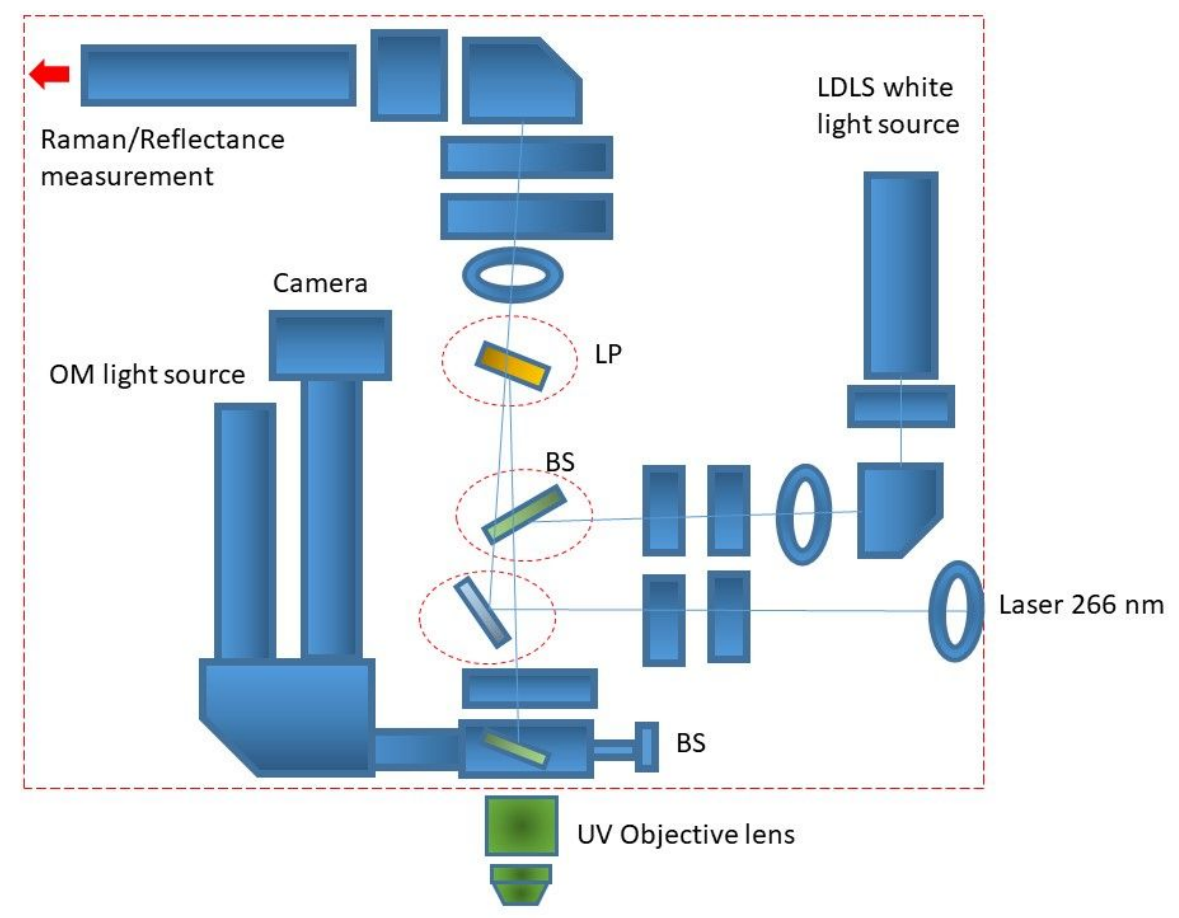

Figure S2 The schematic of home build reflectance/Raman measurement optical setup. 

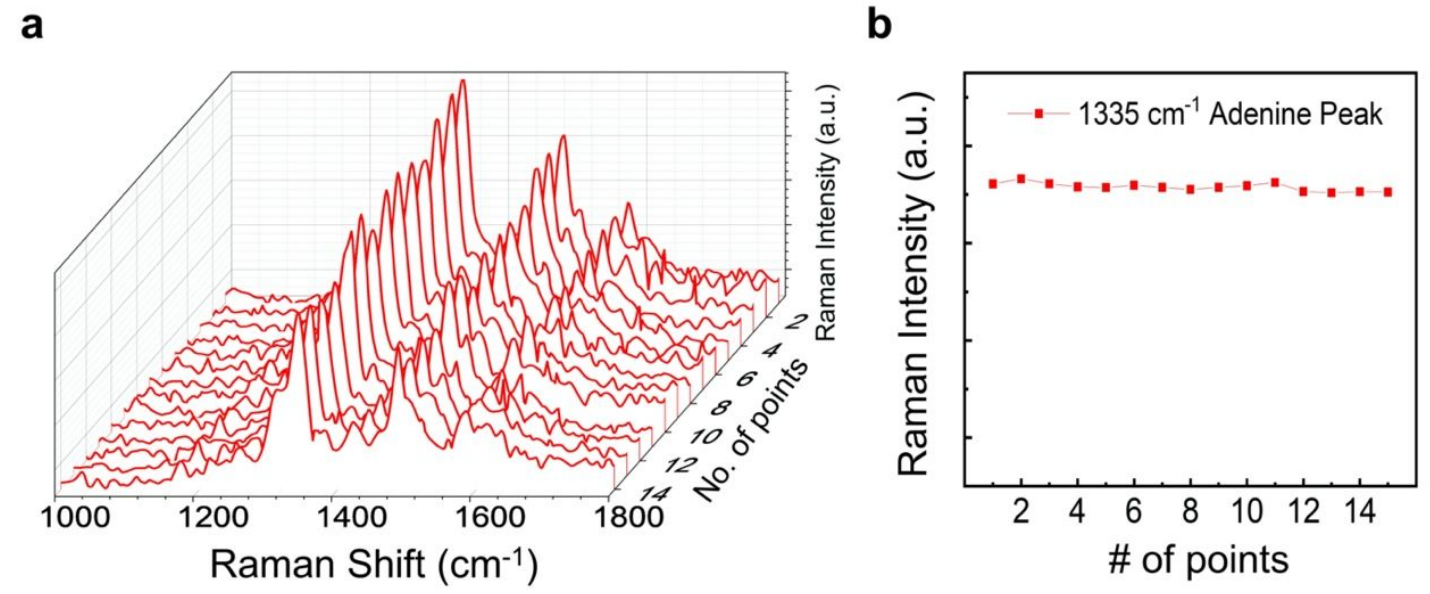

Figure S3 a, The SERRS spectra of $(\mathrm{A})_{12}$ on different 15 points on Al nanohole array. $\mathbf{b}$, Variation of Raman intensity peak of $(\mathrm{A})_{12}$ at $1335 \mathrm{~cm}-1$. 
Reference:

(1) Cheng, C.-W.; Liao, Y.-J.; Liu, C.-Y.; Wu, B.-H.; Raja, S. S.; Wang, C.-Y.; Li, X.; Shih, C.-K.; Chen, L.-J.; Gwo, S. Epitaxial Aluminum-on-Sapphire Films as a Plasmonic Material Platform for Ultraviolet and Full Visible Spectral Regions. ACS Photonics 2018, 5 (7), $2624-2630$.

(2) Jha, S. K.; Ahmed, Z.; Agio, M.; Ekinci, Y.; Löffler, J. F. Deep-UV Surface-Enhanced Resonance Raman Scattering of Adenine on Aluminum Nanoparticle Arrays. J. Am. Chem. Soc. 2012, 134 (4), 1966-1969.

(3) Sigle, D. O.; Perkins, E.; Baumberg, J. J.; Mahajan, S. Reproducible Deep-UV SERRS on Aluminum Nanovoids. J. Phys. Chem. Lett. 2013, 4 (9), 1449-1452.

(4) Ding, T.; Sigle, D. O.; Herrmann, L. O.; Wolverson, D.; Baumberg, J. J. Nanoimprint Lithography of Al Nanovoids for Deep-UV SERS. ACS Appl. Mater. Interfaces 2014, 6 (20), 17358-17363.

(5) Kumamoto, Y.; Taguchi, A.; Honda, M.; Watanabe, K.; Saito, Y.; Kawata, S. Indium for Deep-Ultraviolet Surface-Enhanced Resonance Raman Scattering. ACS Photonics 2014, 1 (7), 598-603.

(6) Sharma, B.; Cardinal, M. F.; Ross, M. B.; Zrimsek, A. B.; Bykov, S. V.; Punihaole, D.; Asher, S. A.; Schatz, G. C.; Van Duyne, R. P. Aluminum Film-Over-Nanosphere Substrates for Deep-UV Surface-Enhanced Resonance Raman Spectroscopy. Nano Lett. 2016, 16 (12), 7968-7973. 